\title{
vICC: Varying Intraclass Correlation Coefficients in $\mathrm{R}$
}

\section{Donald R. Williams ${ }^{1}$}

DOI: $10.21105 /$ joss.0XXXX

1 Department of Psychology, University of California, Davis

\section{Software}

- Review ¿

- Repository ca

\section{Summary}

- Archive ct

Editor: Editor Name ct

In mixed-effects (a.k.a, hierarchical or multilevel) models, intraclass correlation coefficients (ICC) are commonly computed, with applications spanning from characterizing group-level homogeneity (Shieh, 2016) to measurement reliability (Williams et al., 2020). While there are a wide spectrum of applications, an underlying assumption of each is that the variance

Submitted: 01 January $X X X X$ Published: 01 January $X X X X$ components used in their computation are fixed and non-varying. Consider the case of ICC(1), that is,

$$
\operatorname{ICC}(1)=\frac{\sigma_{b}^{2}}{\sigma_{b}^{2}+\sigma_{w}^{2}}
$$

where $\sigma_{b}^{2}$ is the between-group variance and $\sigma_{w}^{2}$ the within-group variance. In a one-way random effects model, $\sigma_{w}^{2}$ is essentially the average within-group variance. However, if there are group-level differences in $\sigma_{w}^{2}$, this implies that there is group-level variation in the ICC.

The methodology in the R package vICC was specifically designed to quantify variation in ICC(1) by allowing $\sigma_{w}^{2}$ to vary. This can be used to indentify groups that are more (or less) homogeneous, as well as which groups are adequately described by Equation (1). There is currently no software for this purpose.

\section{Statement of Need}

The vICC package can be used to:

- Obtain posterior probabilities that each group shares a common within-group variance (i.e., $\sigma_{w}^{2}$ in Equation 1). This is accomplished with the spike and slab approach for Bayesian hypothesis testing (a review is provided in O'Hara \& Sillanpää, 2009).

- Test for between-group differences in $\sigma_{w}^{2}$. This is also accomplished with a spike and slab formulation.

- Compute group-specific ICCs, that is the correlation for any two observations from the same group, and ICC(2), that is average score reliability. Both $\operatorname{ICC}(1)$ and $\operatorname{ICC}(2)$ are reliability indices.

Additionally, there are plotting capabilities using the R package ggplot2 (Wickham, 2016). 


\section{Methodology}

The following models are in vICC:

1. pick_group:

This model has a spike and slab on the random intercepts for the within-group variance. This provides posterior inclusion probabilities (PIP) that each group (e.g., person) does not belong to the common within-group variance model.

2. pick_tau:

This model has a spike and slab on the random effects standard deviation in the scale model which captures between-group variability in the within-group variances. This provides a PIP that there is variation in the within-group variances.

3. pick_none:

This model also provides group-specific reliability, but there is no spike and slab formulation. This is perhaps ideal for those not familiar with Bayesian testing, but would still like to compute varying ICCs.

4. customary:

This is the standard random intercepts model that assumes a common within-group variance.

Note that options 1 and 2 provide Bayesian model averaged estimates for the ICCs. The model formulations are provided in Williams et al. (2019).

\section{Implementation}

The following estimates the pick_group model for reaction times from a cognitive task (Hedge et al., 2018).

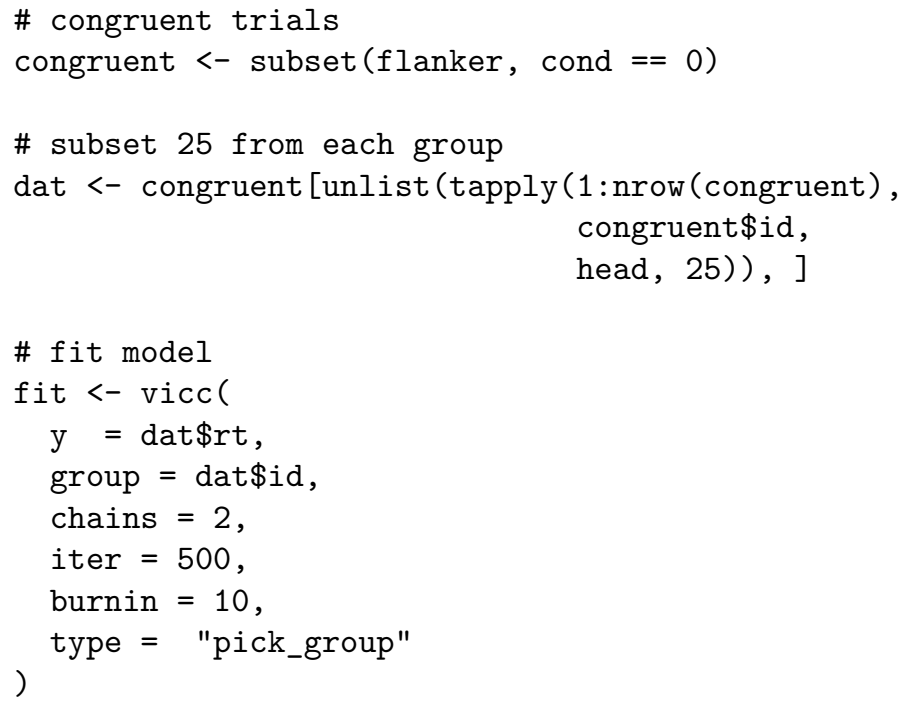

The posterior inclusion probabilities are then plotted with plot (pip(fit)). 


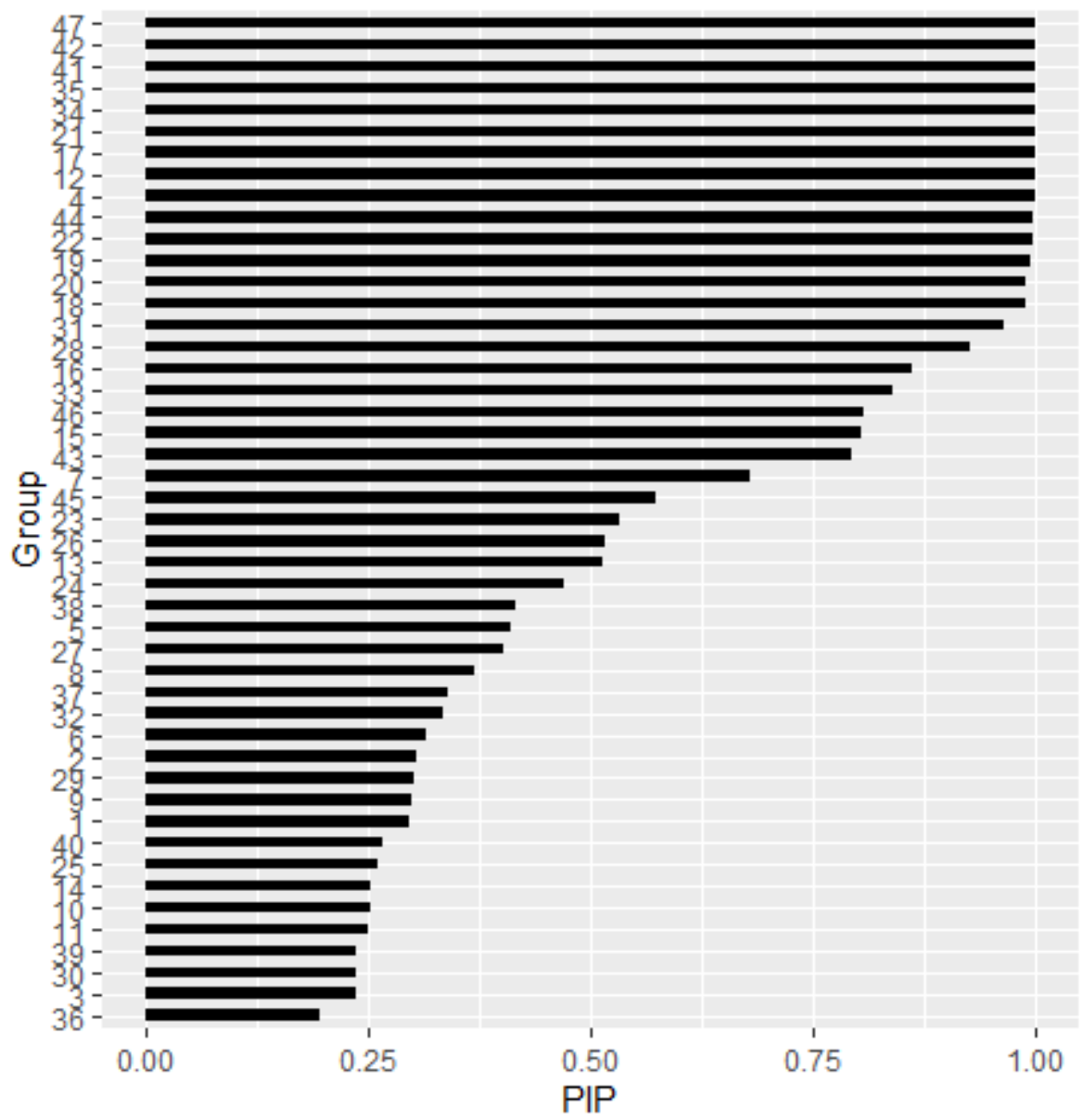

Figure 1: Posterior Inclusion Probabilities

Figure 1: Posterior inclusion probabilities for each group that provide the evidence for differing from the average within-group variance.

The group-level ICCs are plotted with








Figure 2: Group-level intraclass correlation coefficients. The black line is the customary ICC computed with Equation (1).

Notice that the object plts can be further modified with ggplot2. Further, it also includes plots for the means (plot_mean), standard deviations(plot_sd), ICC(2) (plot_icc2).

\section{Conclusion}

The vICC package allows researchers to investigate variability in the intraclass correlation coefficient. In the future, the suite of models will be expanded to allow for partitioning the variance among several grouping variables.

\section{Acknowledgements}

DRW was supported by a National Science Foundation Graduate Research Fellowship under Grant No. 1650042

\section{References}

Hedge, C., Powell, G., \& Sumner, P. (2018). The reliability paradox: Why robust cognitive tasks do not produce reliable individual differences. Behavior Research Methods, 50(3), 1166-1186.

O'Hara, R. B., \& Sillanpää, M. J. (2009). A review of bayesian variable selection methods: What, how and which. Bayesian Analysis, 4(1), 85-118. https://doi.org/10.1214/ 09-BA403

Shieh, G. (2016). Choosing the best index for the average score intraclass correlation coefficient. Behavior Research Methods, 48(3), 994-1003. https://doi.org/10.3758/ s13428-015-0623-y 
Wickham, H. (2016). ggplot2: Elegant Graphics for Data Analysis. Springer-Verlag New York. ISBN: 978-3-319-24277-4

Williams, D. R., Martin, S. R., DeBolt, M., Oakes, L., \& Rast, P. (2020). A fine-tooth comb for measurement reliability: Predicting true score and error variance in hierarchical models. PsyArXiv.

Williams, D. R., Martin, S. R., \& Rast, P. (2019). Putting the individual into reliability: Bayesian testing of homogeneous within-person variance in hierarchical models. PsyArXiv. 